\title{
EDITORIAL
}

\section{Complexity and Healing Relationships}

The Institute of Medicine's first simple rule for quality health care is a long-term healing relationship; and the first aim for a quality health care system is patient-centered care. ${ }^{1}$ The Association of American Medical Colleges' Medical School Objective Project urged faculties to teach interpersonal and communication skills. ${ }^{2}$ The Accreditation Council for Graduate Medical Education accredits residency programs based, in part, on their demonstration of residents' competence in interpersonal and communication skills. ${ }^{3}$ Similarly, the American Board of Medical Specialties' member boards include communication skills in the criteria for certification and recertification. ${ }^{4}$ Yet the scientific and theoretical basis for these recommendations remains sparse.

The proceedings of the conference, Re-Forming Relationships in Health Care: Creating a National Research Agenda for Relationship-Centered Care, published in this supplement contributes to rectifying this deficiency. The plenary papers explain how, why, and what makes healing relationships succeed and fail. The conference outlines an ambitions agenda of research questions about the centerpiece of all health carethe healing relationship. The answers to these questions promise to inform healers, teachers of healers, evaluators of the quality of healing, and social institutions that support healing.

The philosophy of medical care called relationship-centered care (RCC) provides the ideological glue that holds these papers together. ${ }^{5}$ On superficial inspection RCC appears to be a synonym for its antecedent, and possibly more familiar patient-centered care or the humanistic qualities of Care. Such short shrift would be unfortunate, for RCC plows new ground by expanding the concept of the physician patient relationship to one that includes the personhood of both the patient and the physician, acknowledges the central role played by the emotions of both parties in the relationship and the fact that the relationship is shaped by reciprocal influence rather than only by humanistic, but still largely unidirectional, therapeutic ministrations.

Were the RCC philosophy to stop with expanding the ideas about the physician patient relationship, it still would be a substantial advance; however, its authors go further. They promote the idea that health care emerges from the relationships between all of the people who make up the institutions and communities responsible for health care. In all of these relationships, the personhood, emotions, and reciprocal influences of everyone on everyone else actively shape the quality of care, the experience of health and illness, and satisfaction of everyone involved. ${ }^{6}$

At first blush, RCC appears to challenge the basic tenants of traditional medical professionalism. It displaces the old idea that physicians be compassionate yet somewhat depersonal-

Address correspondence and requests for reprints to Dr. Duffy: American Board of Internal Medicine, 510 Walnut Street, Suite 1700, Philadelphia, PA 19010 (e-mail: dduffy@abim.org). ized, and practices medicine with scientific objectivity and skepticism in a humane and altruistic manner with the idea that a physician's humanity, emotions, motives, and capacity to influence and be influenced play a central role in healing relationships.

On still deeper examination the ideology of RCC is very American. It incorporates the key humanistic qualities of compassion, respect, and integrity. It is based on freedom of speech, openness, respect for individual persons, reflective self-awareness, and welcoming diversity and disruption. Relationship-centered care democratizes physician and patient roles and democratizes the roles of everyone in health care systems with the citizens of a society that values health.

Relationships are often thought of as being the medium for establishing trust and communication; and communication is the exchange of information, ideas, feelings, and meanings between at least two persons. Some relationships are based on simple exchange of instrumental information about how to do something. Others involve deeply understanding perspectives, particularly unique, interior and very personal perspectives like the experience of illness. The first type of communication is context thin and lends itself well to written instructions or verbal exchanges. These can be accomplished through asynchronous communications like writing and reading, e-mail, and so on.

The paper by Weiner and Biondich ${ }^{7}$ describes nicely how modern information technology can make low context communication more effective and efficient. In so doing, information technology can enhance trust in the reliability of routine process. However, information technology alone is no substitute for, and may even interfere with, context-rich communication.

Context-rich communication such as that involved in making an ambiguous or complex diagnosis, or in motivating patients to undergo complex and risky treatment, involves deeper understanding of values, meaning, and emotions. Trust is essential in these risky and intensely personal relationships. Roter et al.' ${ }^{8}$ paper explores what is known about context-rich relationships. They describe emotional intelligence as the ability to decode emotions expressed by others, communicate one's own emotions, and to become self-aware of the interplay of one's emotions and motivations with cognitive processes and actions. These abilities shape the essential elements for competence in communication and interpersonal skills. Moreover, these same skills seem to be essential for effective teamwork with colleagues and for influencing leaders of health care organizations and public policy makers.

The relationship between medical teachers and their students and residents shapes the new doctor's core values, beliefs, and habits that make up professionalism. Haidet and Stein $^{9}$ describe various assumptions about professional behavior and values that are transmitted, not through a formal 
curriculum, but an informal one that is embedded in the culture of how senior doctors are observed relating to patients.

Diving deeper into the impact of personhood on healing relationships, Cooper et al. ${ }^{10}$ explore how cultural, ethnic, and socioeconomic differences affect the outcomes and satisfaction of patients and physicians with health care. Our ethnic, cultural, racial, and other identifying qualities seem to impose themselves mindlessly in the physician patient relationship. Relationship-centered care shows how the personhood of individuals, self-awareness of subtle expressions of emotions, subliminal beliefs, and the pernicious effect of pattern recognition (stereotyping when applied to ethnicity or race) for efficient decision making conspire to erode our professional aspiration for equitable care and fair treatment.

I think the most provocative paper of the conference, however, is Suchman's theory of human interaction called Complex Responsive Processes of Relating (CRPR). ${ }^{11}$ It offers strong theoretical confirmation for the principles and practices of RCC. Building upon work of Ralph Stacey and Thomas Smith it incorporates insights from complexity theory, sociology, and social constructionism to show how patterns of meaning and relating are continuously self-organizing in the course of human interactions. It turns our ideas about communication, interpersonal skills, and even professionalism on their head.

Suchman begins by challenging four fundamental assumptions about medical communication and relationship: (1) all behavior in the medical encounter is intentional, (2) behavior in the medical encounter follows linear causality, in that every effect has a cause that is ultimately discoverable, (3) communication is a process of information transfer, and (4) most studies of communication and relationship use the medical encounter in an office visit as the basic unit of observation to correlate presumably static independent variables with outcome variables presumed to be enduring.

In place of these assumptions, his theory proposes that human interaction is nonlinear and characterized by unpredictable and emergent patterns of meaning or patterns of relating that self-organize without anyone's intention or direction. These patterns emerge from the relationship process itself. Words and phrases or nonverbal gestures introduced into dialogue merge with memories, beliefs, judgments, and emotions in each individual. These interior dimensions of the relationship process are invisible to the researching observer. They occur in an internal dialogue within the brain and body of each participant. Of course, everything in an encounter is not chaos. Stable patterns of relating are enacted, like asking routine history questions and recording the answers, but the attendant complexity of each response has the potential for shaping reciprocating ideas and reactions so that a totally unique interaction emerges.

These iterative reciprocal interactions are the hallmark of nonlinear dynamics and form the basis of complexity theory. In complex responsive processes there is no equilibrium state and patterns can shift unpredictably, providing that order and disorder, constraint and freedom co-exist. Nonlinear interactions amplify small differences; for example, in just a few cycles of interaction, the small difference can be amplified into a new, transformative pattern, providing that diversity has been introduced and one party in the relationship is responsive to small changes in the interaction.

When applied to changing the culture of an organization or institution (like the hidden curriculum), the CRPR theory suggests that such change occurs not by designing new structures but by introducing new themes into the organizational conversation in the hope that they will amplify and disseminate. Therefore an effective way to change a pattern would be mindfully enacting the new pattern in each moment to create repeated disturbances in the traditional pattern in the hope that they will amplify and spread. Or in the words of Mahatma Gandhi, "be the change you want to see in the world."

It is not surprising that physicians, in the pursuit of efficiency and reliability, might be inclined to dampen these perturbations through mentally stereotyping culturally different patients or ignoring nuanced emotional signals. Although only hinted at, in this article, the theory also links discoveries in neurobiology and molecular genetic mechanism with the conversations in relationship that not only describe but also shape our human experience of health and disease and the nature of institutional cultures.

One of the most exciting aspects of the CRPR theory is its capacity for explaining the importance of teamwork and partnership that emerges from reciprocal influence and stable patterns of behavior called methods. It seems to be possible to stimulate cultural change in organizations and institutions by deliberately and intentionally introducing new themes or patterns which cause perturbation in stable systems. These perturbations provide the opportunity for novel attractors to coalesce into new patterns that self-organize from old ones.

The reciprocal influence of words, emotions, ideas, memories, and images cannot be overestimated. They are the elemental machinery that creates self-organizing, emergent, and stable patterns of human achievement-through personal behavior, experience of self, and the experience of health or distress and all human social enterprises.

Proof or disproof of the theory through empiric evidence lies ahead. New methodological approaches must be developed. As it has taken over 10 years since the principles of RCC were first articulated until they were used to organize an important gathering of researchers for setting a work agenda, it is likely to take at least that long until the full implications of Complex Responsive Processes Relationship will be fully appreciated.-F. Daniel Duffy, MD, FAAPP, American Board of Internal Medicine, Philadelphia, PA, USA.

\section{REFERENCES}

1. Committee on Quality of Health Care in America, Institute of Medicine. Crossing the Quality Chasm: A New health System for the 21 st Century. Washington, DC: National Academy Press; 2001.

2. Learning objectives for medical student education-guidelines for medical schools: report I of the Medical School Objectives Project. Acad Med. 1999;74:13-8.

3. Available at http://www.acgme.org/acWebsite/navPages/nav_comPR.asp. Accessed October 3, 2005.

4. Assessment of physician-patient communication conference proceedings, March 22-23, 2002. Available at http://www.abms.org./conferences.asp. Accessed October 3, 2005.

5. Tresolini CP and the Pew Fetzer Task Force on Psychosocial Education. Health Professions Education and Relationship-Centered Care: Report of the Pew-Fetzer Task Force on Advancing Psychosicial Education. San Francisco, CA: Pew Health Commission; 1994:1-76.

6. Beach MC, Inui T. Relationship-centered care: a constructive reframing. J Gen Intern Med. 2006;21:S3-8.

7. Weiner M, Biondich P. The influence of information technology on patient-physician relationships. J Gen Intern Med. 2006;21:S35-39. 
8. Roter DL, Frankel RM, Hall JA, Sluyter D. The expression of emotion through nonverbal behavior in medical visits: mechanisms and outcomes. J Gen Intern Med. 2006;21:S28-34.

9. Haidet P, Stein HF. The role of the student-teacher relationship in the formation of physicians: the hidden curriculum as process. J Gen Intern Med. 2006;21:S16-20.
10. Cooper LA, Beach MC, Johnson RL, Inui TS. Delving below the surface: understanding how race and ethnicity influence relationships in healthcare. J Gen Intern Med. 2006;21:S21-27.

11. Suchman AL. A new theoretical foundation for relationshipcentered care: complex responsive processes of relating. J Gen Intern Med. 2006;21:S40-45. 